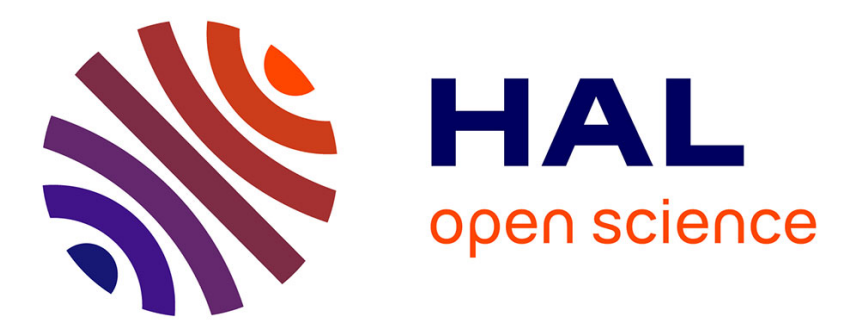

\title{
Adaptive high gain observer based output feedback predictive controller for induction motor
}

\author{
S. Hadj Saïd, F. M'Sahli, F. Mimouni, Mondher Farza
}

\section{To cite this version:}

S. Hadj Saïd, F. M'Sahli, F. Mimouni, Mondher Farza. Adaptive high gain observer based output feedback predictive controller for induction motor. Computers and Electrical Engineering, 2013, 39 (2), pp.Pages 151-163. 10.1016/j.compeleceng.2012.12.016 . hal-01062310

\section{HAL Id: hal-01062310 https://hal.science/hal-01062310}

Submitted on 12 Sep 2014

HAL is a multi-disciplinary open access archive for the deposit and dissemination of scientific research documents, whether they are published or not. The documents may come from teaching and research institutions in France or abroad, or from public or private research centers.
L'archive ouverte pluridisciplinaire HAL, est destinée au dépôt et à la diffusion de documents scientifiques de niveau recherche, publiés ou non, émanant des établissements d'enseignement et de recherche français ou étrangers, des laboratoires publics ou privés. 


\title{
Adaptive high gain observer based output feedback predictive controller for induction motors ${ }^{\text {is }}$
}

\author{
S. Hadj Saïd ${ }^{\mathrm{a}, *}$, F. M'Sahli ${ }^{\mathrm{a}}$, M.F. Mimouni ${ }^{\mathrm{a}}$, M. Farza ${ }^{\mathrm{b}}$ \\ ${ }^{a}$ Department of Electrical Engineering, Monastir Engineering School, Road Ibn Eljazzar, 5019 Monastir, Tunisia \\ ${ }^{\mathrm{b}}$ GREYC, UMR 6072 CNRS, Universite de Caen, 6 Bd Marechal Juin, 14050 Caen Cedex, France
}

\begin{abstract}
A B S T R A C T
An accurate estimation of both rotor and stator resistances is usually required to achieve high performance control in induction machine drive systems. In our study, an output feedback predictive controller which is adaptive with respect to these parameters uncertainties is proposed. Such design attempts to a fully decoupling in speed and flux magnitude when conjoint estimation of the state and the critical parameters is performed. Precisely, twostage of high gain observer are used to provide on-line update of rotor and stator resistances as well as following time evolution of the rotor flux and the load torque. Then, the instantaneous estimates are exploited in a constrained predictive controller for achieving the admissible tracking objective. The little number of the synthesis parameters and the ability of the control scheme to handle input constraints constitute the two main benefits of our design. Simulation results show that the proposed controller is suitable for high dynamics performance applications.
\end{abstract}

\section{Introduction}

High performance control of induction machines (IM) remains a challenge, not only because the strongly coupled nonlinearities and the MIMO nature of motor model; but also, due to the unknown load disturbance and parameters uncertainty. The lack of knowledge of parameters is mostly related to the stator and rotor resistances which are subjected to large variations during operations [1,2]. It is essential to know that in addition to the load torque, the temperature and the frequency dependent variation of the stator and rotor resistances. In particular, the rotor resistance value can increase by an order of $100 \%$, which involves significant undesirable effects on the vector control algorithm [3-5]. In other hand, the stator currents strongly affects the stator resistance due to the ohmic losses in the winding, which presents a serious hindrance when a vector controlled drive operates at low speed regions [6-8]. So, any mismatch between the parameters in the motor and that instrumented in the vector controller will result in the deterioration of performance in terms of steady state error and transient oscillations of the rotor flux and torque $[9,10]$. Consequently, the efficiency of the motor drive decreases and cannot answer to the high precision performance which is usually needed in machine tools or robotics application. All this gives a strong motivation towards the development of adaptive output feedback controller in order to alleviate these performance degradations, and consequently various schemes have been proposed in the literature for simultaneous estimation of the motor resistances and the internal states in IM. In [11], the proposed adaptive controller including an estimation procedure is able to follow very efficiently the actual values of both resistances, but eleven parameters should be tuning. Immersion technique based forgetting factor observer is investigated in [12]. In this case, the authors propose to extend IM model in

\footnotetext{
Reviews processed and approved for publication by Editor-in-Chief Dr. Manu Malek.

* Corresponding author.

E-mail addresses: salim.hadjsaid06@yahoo.fr, salim.hadjsaid@issig.rnu.tn (S. Hadj Saïd).
} 
order to obtain a representation which is affine with respect to the variables to be estimated. Nevertheless, such strategy remain inapplicable when rotor flux states are using in the model. In [13], a direct torque control (DTC) method is used for the tracking objective and a sliding mode technique is adopted for speed and flux estimation as well as the stator resistance adjustment. It is shown that the speed estimator and the resistance adaptation suffer from variations of the rotor resistance and the load torque, respectively. Both motor resistances are estimated in [14] by injecting high frequency signals to the flux and magnetizing current commands. However, the algorithm identifying the resistances used in the feedback linearization controller is applicable only when the control system is in steady state but not when the load torque is varying largely or when the speed command is being changed, as stated by the authors. A development of switching extended Kalman filter (EKF) for rotor and stator resistances in speed sensorless control of IM is introduced in [15,1]. The lack of stability results and the requirement of high computation demand constitute both main drawbacks of the proposed design.

If the problem of IM control has been addressed by a variety of methods such as, field oriented control (FOC), direct torque control (DTC), feedback linearization controller, sliding mode controller (SMO), etc.; nonlinear model predictive control (NMPC) strategies are seldom used in this field, mainly due to their computational burden. Nonetheless, predictive control laws are popular and well established methods in the process industries [16]. An inherent advantage of NMPC methodology consists in its ability to handle constraints and nonlinearity in optimal way [17]. Recently, the so called nonlinear generalized predictive control (NGPC) introduced in [18,19], can constitute a remedy to the computational issue. Indeed, an explicit solution of the state feedback control is given in the continuous time. It arises from the Taylor series approximation of the output tracking error. Moreover, the closed loop stability is guaranteed when the relative degree of system is well definite.

The aim of this paper is to design an output feedback predictive controller for achieving a tracking objective in spite of parameters uncertainty. To this end, two-stage of high gain observer (HGO) are used to accurately determine on-line the internal state variables and the time varying parameters. Thereafter, estimated variables are integrated in a constrained NGPC scheme in order to generate a decision variable that performs angular speed and flux magnitude reference tracking. For achieving the boundedness of the resultant control voltages, input constraints are inherently taking into account when the controller is synthesized.

The remainder of the paper is organized as follows: in Section 2, the mathematical model of induction machine is recalled. We describe in Section 3 the procedure of designing a suitable HGO corresponding to the particular class of nonlinear systems in which, the model previously given should be transformed. In the spirit of NGPC law developed in [19], a synthesis of the output feedback controller is detailed in Section 3. In particular, by using a quadratic programming $(\mathrm{QP})$ or linear matrix inequalities (LMI) formalisms, the control problem is reformulated as a convex optimization problem subject to input constraints. With relevant simulation results, Section 5 illustrates the tracking target performance as well as the parameters adaptation behavior. Concluding remarks are discussed in the final section.

\section{IM model and problem statement}

Assuming linear magnetic circuits, the dynamics of a balanced nonsaturated induction motor in a fixed reference frame attached to the stator are given in a condensed form as follows:

$$
\left\{\begin{array}{l}
i=\mu F\left(\omega, \alpha_{r}\right) \Psi-\gamma\left(\alpha_{r}, \alpha_{s}\right) l+\frac{1}{\sigma L_{s}} u \\
\dot{\Psi}=-F\left(\omega, \alpha_{r}\right) \Psi+M \alpha_{r} l \\
\dot{\omega}=\frac{p M}{J L_{r}} l^{T} J_{2} \Psi-\frac{1}{J} T_{L} \\
y=l
\end{array}\right.
$$

in which $\omega$ is the rotor speed, $\Psi=\left[\Psi_{\alpha r}, \Psi_{\beta r}\right]^{T}$ is the rotor flux vector, $l=\left[l_{\alpha s}, l_{\beta s}\right]^{T}$ and $u=\left[u_{\alpha s}, u_{\beta s}\right]^{T}$ are the stator current and voltage vectors in a fixed reference attached to the stator. The model parameters are: rotor moment of inertia $J$, rotor and stator winding's resistances $\left(R_{r}, R_{s}\right)$ and inductances $\left(L_{r}, L_{s}\right)$, mutual inductance $M$ and load torque $T_{L}$. To simplify notations we use the reparametrization: $\mu=\frac{M}{\sigma L_{r} L_{s}}, \sigma=1-\frac{M^{2}}{L_{r} L_{s}}, \gamma\left(\alpha_{r}, \alpha_{s}\right)=\frac{\alpha_{s}}{\sigma}+M \alpha_{r} \mu, F\left(\omega, \alpha_{r}\right)=\alpha_{r} I_{2}-p \omega J_{2}$, with $\alpha_{r}=\frac{R_{r}}{L_{r}}, \alpha_{s}=\frac{R_{s}}{L_{s}}, I_{2}$ is the 2-dimensional identity matrix and $J_{2}=[0-1 ; 10]$ is a skew-symmetric matrix.

The load torque is considered an unknown bounded disturbance with constant time derivative. Moreover, due to variations of both rotor and stator resistances during operations, $\alpha_{r}$ and $\alpha_{s}$ are assumed to be uncertain within known bounds.

The control objective consists in tracking a smooth predefined profile for the motor speed $\omega$, while regulating the square of rotor flux modulus $\|\Psi\|^{2}$ at a desired constant value. Since both rotor flux and load torque are assumed unaccessible to measure and in presence of rotor and stator resistances mismatch, we should synthesize a fast observer that allows to estimate conjointly these states and parameters, and then incorporated them into the control loop. On the other hand, as it is well known, peaking phenomena can usually occur when combining observer and controller. Precisely, during the transient, the peak of the estimation error often generates a large control action which may drive the closed loop system trajectories to instability. So, such problem should be taking into account when the controller is designed for ensuring the evolution of both states and decision variables trajectories inside a predefined limits. 


\section{Nonlinear class of study and high gain observer design}

For computational issue, the high gain observer which admits an explicit correction gain, is shown as one of the most viable candidate that can resolve the problem of variables estimation in a little time. However, the procedure of HGO is restricted to a particular class of nonlinear systems as the following form:

$$
\left\{\begin{array}{l}
\dot{x}=f(u, s, x)+\varepsilon \\
y=\bar{C} x=x^{1}
\end{array}\right.
$$

where the state $x \in \mathbb{R}^{n}$ with $x^{k} \in \mathbb{R}^{n_{k}}$ for $k=1,2, \ldots, q$, and $n_{1} \geqslant n_{2} \geqslant \cdots, n_{q}, \sum_{k=1}^{n} n_{k}=n, s(t)$ is a known signal, the input $u \subset \mathbb{U}$ a compact set of $\mathbb{R}^{m}$, the output $y \in \mathbb{R}^{n_{1}}$,

$$
\begin{aligned}
& x=\left(\begin{array}{c}
x^{1} \\
x^{2} \\
\vdots \\
x^{q}
\end{array}\right) ; \quad f(u, s, x)=\left(\begin{array}{c}
f_{1}\left(u, s, x^{1}, x^{2}\right) \\
f_{2}\left(u, s, x^{1}, x^{2}, x^{3}\right) \\
\vdots \\
f_{q-1}(u, s, x) \\
0
\end{array}\right) ; \quad \varepsilon=\left(\begin{array}{c}
0 \\
\vdots \\
0 \\
\varepsilon^{q-1} \\
\varepsilon^{q}
\end{array}\right) \\
& \bar{C}=\left[\begin{array}{llll}
I_{n_{1}}, & 0_{n_{1} \times n_{2}}, \ldots, & 0_{n_{1} \times n_{q}}
\end{array}\right]
\end{aligned}
$$

with $I_{n_{1}}$ is the $n_{1} \times n_{1}$ identity matrix and $0_{n_{1} \times n_{j}}$ is the $n_{1} \times n_{j}$ null matrix, $j \in\{2, \ldots, q-1\}$.

$\varepsilon^{k} \in \mathbb{R}^{n_{k}}, k \in\{q-1, q\}$; each $\varepsilon^{k}$ is an unknown bounded real valued function that depend on uncertain parameters. Here, specifying this disturbance term, we propose a slight modification in relation to the model used in [20], when authors choose $\varepsilon$ with only the last component $\varepsilon^{q} \neq 0$. Moreover, as mentioned in [21], the addition of the known signal $s(t)$ do not affect the estimation error convergence.

The synthesis of the HGO corresponding to the form (2), requires to make some assumptions as follows:

(i) There exist $\alpha, \beta$ with $0<\alpha \leqslant \beta$ such that for all $k \in\{1, \ldots, q-1\}, \forall x \in \mathbb{R}^{n}, u \in \mathcal{U}$

$$
0<\alpha^{2} I_{n_{k}} \leqslant\left(\frac{\partial f_{k}\left(u, s, x^{1: k}\right)}{\partial x^{k+1}}\right)^{T} \frac{\partial f_{k}\left(u, s, x^{1: k}\right)}{\partial x^{k+1}} \leqslant \beta^{2} I_{n_{k}} ; \quad \text { with } x^{1: k}=x^{1}, x^{2}, \ldots, x^{k}
$$

Moreover, we assume that $\operatorname{Rank}\left(\frac{\partial f_{k}\left(u, s, x^{1: k}\right)}{\partial x^{k+1}}\right)=n_{k+1}$.

(ii) The function $f(u, s, x)$ is globally Lipchitz with respect to $x$, uniformly in $u$.

(iii) The function $\varepsilon^{q-1}(t)$ and $\varepsilon^{q}(t)$ are uniformly bounded with

$$
\delta_{q-1}=\sup _{t \geqslant 0}\left\|\varepsilon^{q-1}(t)\right\| \text { and } \delta_{q}=\sup _{t \geqslant 0}\left\|\varepsilon^{q}(t)\right\|
$$

By assumption (i), each of $\frac{\partial f_{k}(.)}{\partial x^{k+1}}$ is left invertible. Assumption (ii) can be omitted in the case where the trajectory $x(t)$ of system (2) lie in the bounded set $\Omega$. Whereas assumption (iii) is often physically true because $\varepsilon$ represents the model error.

A candidate HGO for system (2) is described by the following dynamical system:

$$
\dot{\hat{x}}=f(u, s, \hat{x})-\left(\begin{array}{c}
\theta \mathcal{C}_{1}^{q} I_{n_{1}} \\
\theta^{2} \mathcal{C}_{2}^{q}\left[\frac{\partial f_{1}}{\partial x^{2}}(u, s, x)\right]^{+} \\
\vdots \\
\theta^{q} \mathcal{C}_{q}^{q}\left[\prod_{i=1}^{q-1} \frac{\partial f_{k}}{\partial x^{k+1}}(u, s, x)\right]^{+}
\end{array}\right) \bar{C}(\hat{x}-x)
$$

where

- $\theta>0$ is a real number representing the only design parameter of the observer.

- $\mathcal{C}_{j}^{i}=\frac{j !}{i !(j-i) !}$ for $1 \leqslant i, j \leqslant q$; and [.] $]^{+}$is the left inverse of matrix [.].

When we use the observer under form (3) and after a coordinate transformation $\mathfrak{z}=\varrho(x)$, the estimation error is bounded with the following inequality:

$$
\|\tilde{z}(t)\| \leqslant \lambda_{\theta} e^{-\mu_{\theta} t}\|\tilde{z}(0)\|+M_{q-1, \theta} \delta_{q-1}+M_{q, \theta} \delta_{q}
$$

with $\lambda_{\theta}=\sqrt{\frac{\lambda_{\max (s)}}{\lambda_{\min }(s)}} \theta^{q-1}>0, \delta_{q-1}$ and $\delta_{q}$ given in assumption (iii) are the upper bound of $\varepsilon^{q-1}$ and $\varepsilon^{q}$ respectively. $\lim _{\theta \rightarrow \infty} \mu_{\theta}=+-$ $\infty, \lim _{\theta \rightarrow \infty} M_{q, \theta}=0$ and $\lim _{\theta \rightarrow \infty} M_{q-1, \theta}<\mu_{q-1}$. The complete convergence proof is detailed in our previous work (see [22]). It 
should be emphasized that here, the implementation of the high gain observer is quite simple. Indeed, it requires only the tuning of the parameter $\theta$, which should be chosen as a compromise between fast convergence and satisfactory dealing with noise rejection.

\section{Output feedback predictive controller design}

The conjoint states and parameters instantly provided by the observer should be incorporated in the control loop. For achieving an admissible tracking objective, predictive control strategy is preferred not only because it uses few parameters ${ }^{1}$ but also, due to its capability to handle input constraints. This last advantage helps to avoid the peak of the observer estimation error during transient that can generate a large control action driving the closed loop system trajectories to instability. At the next, in the spirit of the state NGPC design given in [19], we develop a constrained output feedback controller that can efficiently applied to the IMs.

\subsection{Predictive control law formulation}

To synthesize the state feedback controller, we shall transform model (2) to a new coordinates for which the design is more easer. In general, some hypotheses are required. Let at this step the following assumptions (iv) There exists a Lipschitz diffeomorphism $\Phi=: \mathbb{R}^{n} \rightarrow \mathbb{R}^{n}, x \mapsto \Phi(x)=\left(\begin{array}{c}\Phi_{z}(x) \\ \Phi \xi(x)\end{array}\right)$ that puts the model (2) under the follow-
ing form

$$
\left\{\begin{array}{l}
\dot{z}=A z+B(b(\xi, z) u+g(\xi, z))+\varphi(z) \\
\dot{\xi}=\eta(\xi, z, u) \\
y=C z
\end{array}\right.
$$

with

$$
\begin{aligned}
& A=\left[\begin{array}{cc}
0 & \bar{A} \\
0 & 0
\end{array}\right], \quad \bar{A}=\text { blockdiag }\left(A_{1}, A_{2}, \ldots, A_{r-1}\right) \\
& B=\left[0_{n_{1}}, \ldots, 0_{n_{1}}, I_{n_{1}}\right]^{T}, \quad z=\left[z^{1^{T}}, z^{2^{T}}, \ldots, z^{r^{T}}\right]^{T} \\
& \varphi(z)=\left[\varphi^{1^{T}}\left(z^{1}\right), \varphi^{2^{T}}\left(z^{1}, z^{2}\right), \ldots, \varphi^{(r-1)^{T}}\left(z^{1}, \ldots, z^{r-1}\right), 0_{n_{1}}\right]^{T}
\end{aligned}
$$

where $z$ and $\xi$ are the internals states of $\operatorname{system}\left(z \in \mathcal{Z} \subset \mathbb{R}^{r n_{1}}\right.$ and $\left.\xi \in \mathbb{R}^{n-m_{1}}\right)$; the control input $u \in \mathcal{U} \subset \mathbb{R}^{m}$; measured output $y \in \mathbb{R}^{n_{1}}$ and $r$ is the relative degree of system $(r \leqslant q)$ in the new coordinates. $g(\xi, z) \in \mathbb{R}^{n_{1}}$ and $b(\xi, z)$ is a rectangular matrix of dimension $n_{1} \times m$ with $n_{1} \leqslant m$. $C$ is as given above, $A_{k} \in \mathbb{R}^{n_{k} \times n_{k}}$ for $k \in\{1, \ldots, r-1\}$ are invertible matrices. They can be obtained from successive $z$ derivatives.

(v) The function $\varphi($.$) is globally Lipschitz in z$ and the functions $b($.$) and g($.$) are globally Lipschitz in z$ uniformly in $\xi$. Moreover, the matrix $b(\xi, z)$ should be of full row rank.

(vi) The system:

$$
\dot{\xi}=\eta \xi, v_{1},\left(\boldsymbol{b}\left(\xi, v_{1}\right)\right)^{+}\left(v_{2}-g\left(\xi, v_{3}\right)\right)
$$

with input $\left(v_{1}, v_{2}, v_{3}\right)$ and state $\xi$ are Input-to-State Stable where $\eta^{+}$denotes the right inverse of $\eta$.

Assumption (vi) is required for ensuring the stability of the system by designing a partial state feedback law. While (iv) and (v), as we will show in section 5.2, are satisfied in the case of induction motors model.

Taking into account the structure of system (5), it is possible to derive the subsystem state trajectory $z_{d}(t) \in \mathbb{R}^{r n_{1}}$ and the associated input sequence $u_{d}(t)$ corresponding to the desired trajectory $z_{d}^{1}(t)$. This allows to define an admissible reference model as follows:

$$
\dot{z}_{d}=A z_{d}+B\left(b\left(\xi, z_{d}\right) u_{d}+g\left(\xi, z_{d}\right)\right)+\varphi\left(z_{d}\right)
$$

where

$$
z_{d}=\left[z_{d}^{1^{T}}, z_{d}^{2^{T}}, \ldots, z_{d}^{r^{T}}\right]^{T} \in \mathbb{R}^{r n_{1}} \text { is the reference state model. }
$$

According to (7), we can extract the state reference trajectory $z_{d}^{k} \in \mathbb{R}^{n_{1}}, k=2, \ldots, r$, as well as the desired control $u_{d} \in \mathbb{R}^{m}$, we have:

\footnotetext{
${ }^{1}$ As we will see $T_{p}$ is the sole design parameter that needs to be adjusted in our control design.
} 


$$
\left\{\begin{array}{l}
z_{d}^{k}=A_{k-1}^{-1} \dot{z}_{d}^{k-1}-\varphi^{k-1}\left(z_{d}^{1}, \ldots, z_{d}^{k-1}\right) \\
u_{d}=\left(b\left(\xi, z_{d}\right)\right)^{+}\left(z_{d}^{r}-g\left(\xi, z_{d}\right)\right)
\end{array}\right.
$$

The proposed state feedback predictive controller is based on the resolution of an optimization problem, which depends on the predicted tracking error as follows [19]:

$$
\mathfrak{I}=\frac{1}{2} \int_{0}^{T_{p}}\left(\bar{y}(t+\tau)-\bar{y}_{d}(t+\tau)\right)^{T}\left(\bar{y}(t+\tau)-\bar{y}_{d}(t+\tau)\right) d \tau
$$

where $T_{p}$ is the predictive period, and $\bar{y}_{d} \in \mathbb{R}^{n_{1}}$ is the prescribed tracking reference.

When assuming that the output $\bar{y}$ and the reference signal $\bar{y}_{d}$ are sufficiently many times continuously differentiable with respect to time $t$, the outputs $\bar{y}(t+\tau)$ and $\bar{y}_{d}(t+\tau)$ at the time $\tau$ are approximately predicted by their Taylor-series expansion up to order $r$ as follows:

$$
\begin{aligned}
& \bar{y}(t+\tau) \doteq \mathcal{T}(\bar{\tau}) Y(t) \\
& \bar{y}_{d}(t+\tau) \doteq \mathcal{T}(\bar{\tau}) Y_{d}(t)
\end{aligned}
$$

where

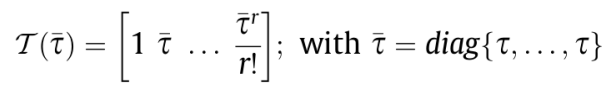

$$
\begin{aligned}
& Y(t)=\left[\begin{array}{llll}
y^{T}(t) & \dot{y}^{T}(t) & \ldots & y^{[r]^{T}}(t)
\end{array}\right]^{T} \\
& Y_{d}(t)=\left[\begin{array}{llll}
y_{d}^{T}(t) \dot{y}_{d}^{T}(t) & \ldots & y_{d}^{[r]^{T}}(t)
\end{array}\right]^{T}
\end{aligned}
$$

Hence, $\Im$ can be rewritten in matrix form as follows:

$$
\mathfrak{I}=\frac{1}{2}\left[Y(t)-Y_{d}(t)\right]^{T} \overline{\mathcal{T}}\left(\bar{T}_{p}\right)\left[Y(t)-Y_{d}(t)\right]
$$

where

$$
\overline{\mathcal{T}}\left(\bar{T}_{p}\right)=\int_{0}^{T_{p}} \mathcal{T}^{T}(\bar{\tau}) \mathcal{T}(\bar{\tau}) d \tau
$$

The $i j$ th elements of the square matrix $\overline{\mathcal{T}}$ are in the form

$$
\overline{\mathcal{T}}_{i j}\left(\bar{T}_{p}\right)=\frac{\bar{T}_{p}^{i+j-1}}{(i+j-1)(i-1) !(j-1) !}
$$

with $\bar{T}_{p}=\operatorname{diag}\left\{T_{p}, \ldots, T_{p}\right\} ; i, j=\{1, \ldots, r+1\}$.

Repeated differentiation up to $r$ times of the output $y_{d}$ with respect to time, gives

$$
\left\{\begin{array}{l}
y_{d}=z_{d}^{1} \\
\dot{y}_{d}=A_{1} z_{d}^{2}+\varphi^{(1)[0]}\left(z_{d}^{1}\right) \\
\ddot{y}_{d}=\left(\prod_{i=1}^{2} A_{i}\right) z_{d}^{3}+\sum_{j=1}^{2}\left(\prod_{i=1}^{2-j} A_{i}\right) \varphi^{(3-j)[j-1]}\left(z_{d}^{1}, z_{d}^{2}\right) \\
\vdots \\
y_{d}^{[r]}=\left(\prod_{i=1}^{r-1} A_{i}\right)\left(b\left(z_{d}, \xi\right) u_{d}+g\left(z_{d}, \xi\right)\right)+\sum_{j=1}^{r-1}\left(\prod_{i=1}^{r-1-j} A_{i}\right) \varphi^{(r-j)[j]}(.)
\end{array}\right.
$$

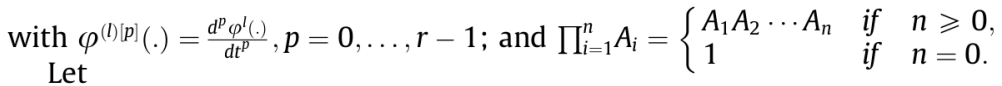

$$
Y(t)-Y_{d}(t)=M\left(z, z_{d}, \xi\right)+\left[\begin{array}{c}
0_{(r-1) n_{1}} \\
H(z, \bar{u}, \xi)
\end{array}\right]
$$

with 


$$
M\left(z, z_{d}, \xi\right)=\left(\begin{array}{l}
e_{z}^{1} \\
A_{1} e_{z}^{2}+d \varphi^{(1)[0]}\left(z^{1}, z_{d}^{1}\right) \\
\left(\prod_{i=1}^{2} A_{i}\right) e_{z}^{3}+\sum_{j=1}^{2}\left(\prod_{i=1}^{2-j} A_{i}\right) d \varphi^{(3-j) \mid j-1]}(.) \\
\vdots \\
\left(\prod_{i=1}^{r-1} A_{i}\right) e_{z}^{r}+\sum_{j=1}^{r-1}\left(\prod_{i=1}^{r-1-j} A_{i}\right) d \varphi^{(r-j)\langle j-1]}(.) \\
-\left(\prod_{i=1}^{r-1} A_{i}\right)\left(b\left(z_{d}, \xi\right) u_{d}+g\left(z_{d}, \xi\right)-g(z, \xi)\right) \\
\quad+\sum_{j=1}^{r-1}\left(\prod_{i=1}^{r-1-j} A_{i}\right) d \varphi^{r-j[j]}(.)
\end{array}\right)
$$

and

$$
H(z, \bar{u}, \xi)=\left(\prod_{i=1}^{r-1} A_{i}\right) b(z, \xi) \bar{u}
$$

such that

$$
\begin{aligned}
& e_{z}^{j}=z^{j}-z_{d}^{j} \text { for } j \in\{1, \cdots, r-1\} \\
& d \varphi^{(j) p p}\left(z^{1}, \ldots, z^{j}, z_{d}^{1}, \ldots, z_{d}^{j}\right)=\frac{d^{p} \varphi^{j}\left(z^{1}, \ldots, z^{j}\right)}{d t^{p}}-\frac{d^{p} \varphi^{j}\left(z_{d}^{1}, \ldots, z_{d}^{j}\right)}{d t^{p}}
\end{aligned}
$$

A simple computation imply that (9) can be rewritten as follows:

$$
\Im\left(\bar{u}, T_{p}\right)=\frac{1}{2}\left(M^{T} \overline{\mathcal{T}} M+2 M^{T} \overline{\mathcal{T}}_{r+1} H(\bar{u})+H^{T}(\bar{u}) \overline{\mathcal{T}}_{(r+1, r+1)} H(\bar{u})\right)
$$

where $\overline{\mathcal{T}}_{r+1}$ is the $r+1$ th column of matrix $\overline{\mathcal{T}}(T)$ and $\overline{\mathcal{T}}_{(r+1, r+1)}$ its $(r+1, r+1)$ th element.

\subsection{Input constrained predictive controller}

In order to achieve the separation principle as given in [23], beside the requirement to use fast dynamic observer (which is solved by choosing $\theta$ so large in the HGO design), we should ensure the boundness of the control signal to avoid peaking phenomena for estimated state. Hence, we need to formulate the control law as a convex optimization problem subject to input constraints.

Note that according to (16), the performance criterion can be rewriting again as follows

$$
\mathfrak{\Im}\left(\bar{u}, T_{p}\right)=\frac{1}{2}\left(M^{T} \overline{\mathcal{T}} M+2 M^{T} \overline{\mathcal{T}}_{r+1}\left(\prod_{i=1}^{r-1} A_{i}\right) b(\hat{z}, \xi) \bar{u}+\bar{u}^{T} b^{T}(\hat{z}, \xi)\left(\prod_{i=1}^{r-1} A_{i}\right)^{T} \overline{\mathcal{T}}_{(r+1, r+1)}\left(\prod_{i=1}^{r-1} A_{i}\right) b(\hat{z}, \xi) \bar{u}\right)
$$

\subsubsection{Proposition 1}

Given a system (5) satisfying assumptions (v) and (vi), an appropriate solution of the output feedback predictive control law which minimizes the cost function (9), arises from a constrained quadratic programming (QP) algorithm as the following form

$$
\begin{array}{ll}
\min _{\bar{u}} & \frac{1}{2} \bar{u}^{T} Q \bar{u}+f \bar{u} \\
\text { subject to } & A_{c} \bar{u} \leqslant B_{c}
\end{array}
$$

where

$$
\begin{aligned}
& Q=b^{T}(\hat{z}, \xi)\left(\prod_{i=0}^{r-1} A_{i}\right)^{T} \overline{\mathcal{T}}_{(r+1, r+1)}\left(\prod_{i=0}^{r-1} A_{i}\right) b(\hat{z}, \xi) \\
& f=M^{T} \overline{\mathcal{T}}_{r+1}\left(\prod_{i=1}^{r-1} A_{i}\right) b(\hat{z}, \xi) \\
& A_{c}=\left[I_{m}-I_{m}\right]^{T} \in \mathbb{R}^{2 m \times m}, \quad B_{c}=\left[U_{\max }^{T}-U_{\min }^{T}\right]^{T} \in \mathbb{R}^{2 m} \\
& U_{\max }=\left[u_{\max }, \ldots, u_{\max }\right]^{T}, \quad U_{\min }=\left[u_{\min }, \ldots, u_{\min }\right]^{T}
\end{aligned}
$$

with $\hat{z}=\Phi(\hat{x})$ is the estimate of $z=\Phi(x)$. 


\subsubsection{Proposition 2}

At the same, we can adopt the LMI formalism for generating the decision variable. Indeed, according to the Schur lemma, the control signal is obtained from the following optimization problem

$\min \kappa \in \mathbb{R}_{\geqslant 0}$

$$
\begin{gathered}
\text { subject to }\left[\begin{array}{cc}
f \bar{u}+M^{T} \overline{\mathcal{T}} M-\kappa & \bar{u}^{T} \\
\bar{u} & -Q^{-1}
\end{array}\right]<0 \\
\left(I_{m} \bar{u}-U_{\max }\right)<0 \\
\left(-I_{m} \bar{u}+U_{\min }\right)<0
\end{gathered}
$$

where $\kappa$ is a small scalar to be minimized.

\subsection{Example for avoiding peaking phenomenon by the constrained predictive controller}

In order to illustrate the efficiency of the proposed control scheme, we attempt to design an output feedback controller for stabilizing around the origin the following system [24]

$$
\left\{\begin{array}{l}
\dot{x}_{1}=x_{2} \\
\dot{x}_{2}=x_{2}^{2}+e^{\left(-x_{2}^{2}\right)} u \\
y=x_{1}
\end{array}\right.
$$

It is clear that the system (20) is under form (2). In accordance to (3), a correspondent HGO is given by

$$
\left\{\begin{array}{l}
\dot{\hat{x}}_{1}=\hat{x}_{2}+2 \theta\left(y-\hat{x}_{1}\right) \\
\dot{\hat{x}}_{2}=\hat{x}_{2}^{2}+e^{\left(-\hat{x}_{2}^{2}\right)} u+\theta^{2}\left(y-\hat{x}_{1}\right)
\end{array}\right.
$$

for which a globally stabilizing controller can easily founded as follows:

$$
u=-e^{\left(\hat{x}_{2}^{2}\right)}\left(\hat{x}_{2}^{2}+\hat{x}_{1}+\hat{x}_{2}\right)
$$

Unfortunately, in spite of using a convergent observer (21) and a stable state feedback (22), the closed loop stability is not achieved when $\theta$ is so large (see Fig. 1, dotted signal). To avoid the peaking of states arising from the estimation error at the transient (when using the same observer (21)), a constrained QP solution can be obtained by (18) with

$$
\begin{aligned}
& Q(\hat{x})=\frac{T_{p}^{5}}{20} e^{\left(-2 \hat{x}_{2}^{2}\right)} \\
& f(\hat{x})=\left(\frac{T_{p}^{3}}{6} \hat{x}_{1}+\frac{T_{p}^{4}}{8} \hat{x}_{2}+\frac{T_{p}^{5}}{20} \hat{x}_{2}^{2}\right) e^{\left(-\hat{x}_{2}^{2}\right)} \\
& A_{c}=\left[\begin{array}{ll}
1 & -1
\end{array}\right]^{T}, b_{c}=u_{\max }\left[\begin{array}{ll}
1 & 1
\end{array}\right]^{T}
\end{aligned}
$$

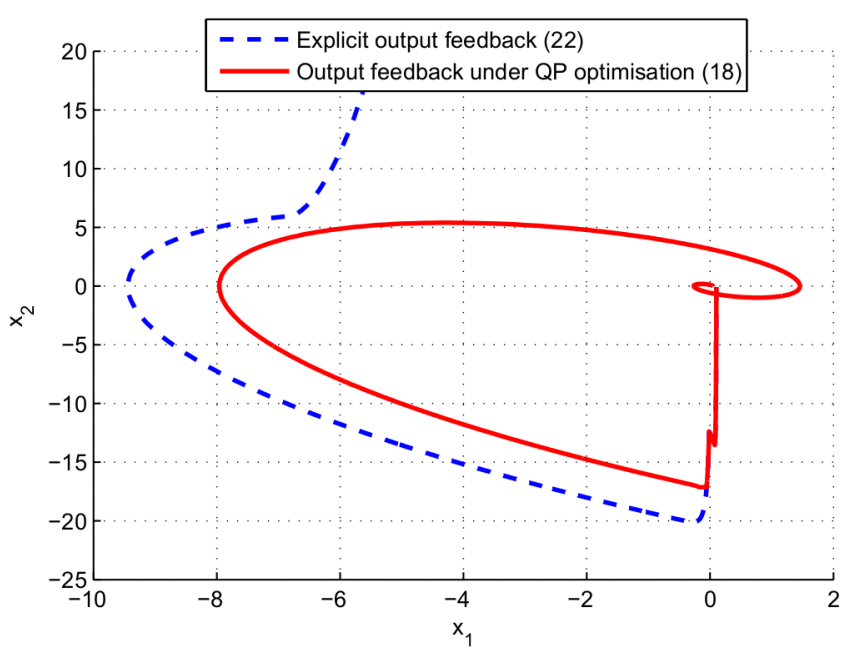

Fig. 1. Ability of input constrained output feedback predictive control to avoid peaking phenomenon. 
Table 1

Induction motor parameters used in the simulations.

\begin{tabular}{llllllll}
\hline$p$ & $f(\mathrm{~Hz})$ & $L_{S}(\mathrm{H})$ & $L_{r}(\mathrm{H})$ & $M(\mathrm{H})$ & $R_{S N}(\Omega)$ & $R_{r N}(\Omega)$ & $J\left(\mathrm{~kg} \mathrm{~m}^{2}\right)$ \\
\hline 2 & 50 & 0.464 & 0.464 & 0.4417 & 5.717 & 3 & 0.0049 \\
\hline
\end{tabular}

Reporting to Fig. 1, it is shown that only when input constraints are taking into account in the control law formulation, the state trajectory $x(t)$ converges asymptotically to the origin $x=0$.

\section{Simulation results for an application to induction motors}

To examine practical usefulness, the proposed estimation algorithm has been simulated for a three-phase $1.5 \mathrm{~kW}$ induction motor, whose rated parameters are depicted in Table 1. Obviously, without measurements of the rotor fluxes and in presence of load torque variations and both rotor and stator resistances uncertainties, the main goal is to control the angular speed and the square of the flux vector norm around the predefined reference trajectories.

\subsection{Estimation of the rotor fluxes, both rotor and stator resistances, and the load torque}

In order to simultaneously estimate both rotor and stator resistances, we use a state transformation such as model (1) can be rewritten under the form (2). Thus, let us definite the new coordinate $x$ such as

$$
\left\{\begin{array}{l}
x^{1}=l \\
x^{2}=\mu\left(F\left(\omega, \alpha_{r}\right) \Psi-\alpha_{r} M l\right)-\frac{1}{\sigma} \alpha_{s} l \\
x^{3}=\left[\begin{array}{ll}
\alpha_{s} & \alpha_{r}
\end{array}\right]^{T}
\end{array}\right.
$$

and $s=\omega$.

Using this transformation, we can derive from model (1) the following submodel

$$
\left\{\begin{array}{l}
\dot{x}^{1}=x^{2}+\frac{1}{\sigma L_{s}} u \\
\dot{x}^{2}=-\frac{1}{\sigma}\left(\dot{x}^{1}+F\left(s, x_{2}^{3}\right) x^{1}\right) x_{1}^{3}-\left(\mu M \dot{x}^{1}\right) x_{2}^{3}-F\left(s, x_{2}^{3}\right) x^{2}+\varepsilon^{2} \\
\dot{x}^{3}=\varepsilon^{3} \\
y=x^{1}
\end{array}\right.
$$

where $\varepsilon^{2}=-\dot{\omega} J_{2} \Psi$. This term shall be treated as unknown bounded function. ${ }^{2}$ We can easily show that the submodel (24) is under form (2). Hence, a corresponding HGO is given by

$$
\left\{\begin{array}{l}
\dot{\hat{x}}^{1}=\hat{x}^{2}+\frac{1}{\sigma L_{s}} u-3 \theta_{1} \tilde{x}^{1} \\
\dot{\hat{x}}^{2}=\underbrace{-\frac{1}{\sigma}\left(\dot{\hat{x}}^{1}+F\left(s, \hat{x}_{2}^{3}\right) \hat{x}^{1}\right) \hat{x}_{1}^{3}-\left(\mu M \dot{\hat{x}}^{1}\right) \hat{x}_{2}^{3}-F\left(s, \hat{x}_{2}^{3}\right) \hat{x}^{2}}_{f_{2}(u, s, \hat{x})}-3 \theta_{1}^{2} \tilde{x}^{1} \\
\dot{\hat{x}}^{3}=-\theta_{1}^{3}\left(\frac{\partial f_{2}(u, s, \hat{x})}{\partial x^{3}}\right)^{+} \tilde{x}^{1}
\end{array}\right.
$$

with

$$
\frac{\partial f_{2}(u, s, \hat{x})}{\partial x^{3}}=\left(\begin{array}{c}
-\frac{1}{\sigma}\left(\dot{\hat{x}^{1}}-p s J_{2} \hat{x}^{1}+\hat{x}^{1} \hat{x}_{2}^{3}\right)^{T} \\
-\left(\mu M \dot{\hat{x}^{1}}+\hat{x}^{2}+\frac{1}{\sigma} \hat{x}^{1} \hat{x}_{1}^{3}\right)^{T}
\end{array}\right)
$$

Referring to (23), we can deduce an estimation of the rotor flux vector and both rotor and stator resistances such as

$$
\left\{\begin{array}{l}
\widehat{\Psi}=F\left(s, \hat{x}_{2}^{3}\right)^{-1}\left(\frac{1}{\mu}\left(\hat{\chi}^{2}+\frac{1}{\sigma} \hat{x}^{1} \hat{x}_{1}^{3}\right)+M \hat{x}_{2}^{3} \hat{x}^{1}\right) \\
\widehat{R}_{r}=L_{r} \hat{x}_{2}^{3} \\
\widehat{R}_{s}=L_{s} \hat{x}_{1}^{3}
\end{array}\right.
$$

Now, rotor fluxes and rotor resistance provided by (25) and (26) are used to determinate the load torque $\widehat{T}_{L}$ and its time derivative $\widehat{T}_{L p}$. So, a second HGO, placed in cascade with the first one, can be designed as follows:

\footnotetext{
${ }^{2}$ If this expression is assumed negligible in [3], in our case it cannot strongly affect the convergence of estimation error when $\theta$ is chosen so large.
} 


$$
\left\{\begin{array}{l}
\dot{\hat{\omega}}=-\frac{1}{J} \widehat{T}_{L}+\frac{p M}{J_{L}} \hat{l}^{T} J_{2} \widehat{\Psi}-3 \theta_{2} \tilde{\omega} \\
\dot{\widehat{T}}_{L}=\widehat{T}_{L p}+3 \theta_{2}^{2} J \tilde{\omega} \\
\dot{\hat{T}}_{L p}=\theta_{2}^{3} J \tilde{\omega}
\end{array}\right.
$$

where $\tilde{\omega}=\hat{\omega}-\omega$.

In order to highlight the features of the adaptive HGO design, besides its privilege in time computation and in the number of synthesis parameters, it is compared with the standard EKF algorithm. Simulation results for conjoint state and parameters estimation of both techniques are illustrated in Fig. 2. We remark that the EKF approach can only estimate constant value of parameters (we show that the estimates cannot track well the real value of parameters when these last change value during operating). However, a real time update of both resistance is achieved when adaptive HGO is used. Moreover, we can ensure that the estimation error converge to zero when the steady state regime is established. Note that recently, to alleviate the estimation error when parameters change during operating, a so called "Switching EKF" alternative is given in [1], but the lack of stability results constitutes the main drawback of such design.

\subsection{Output feedback predictive controller design}

In order to deal with the predictive controller planned above, we shall introduce a mild change of coordinate that allows to put system (1) in the canonical form (5). We use the following intuitive transformation of state

$$
\Phi: \mathbb{R}^{5} \rightarrow \mathbb{R}^{5}, \quad \hat{x}=\left(\begin{array}{c}
\hat{\imath} \\
\hat{\Psi} \\
\hat{\omega}
\end{array}\right) \mapsto\left(\begin{array}{c}
z^{1} \\
z^{2} \\
\xi
\end{array}\right)
$$

with $z^{1}=\left[z_{1}^{1}, z_{2}^{1}\right]^{T}$ and $z^{2}=\left[z_{1}^{2}, z_{2}^{2}\right]^{T}$, such as

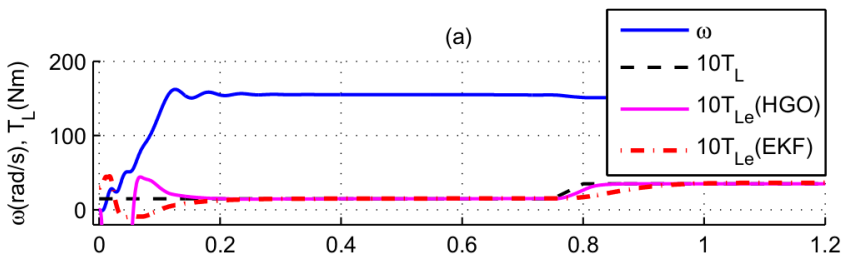

(b)

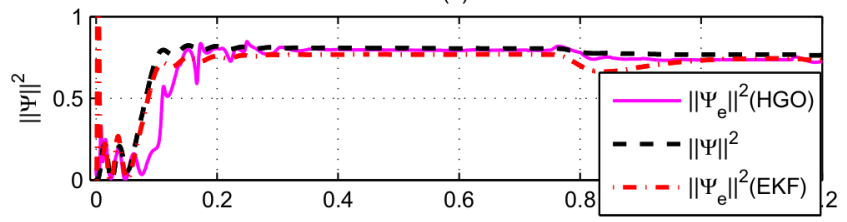

(c)

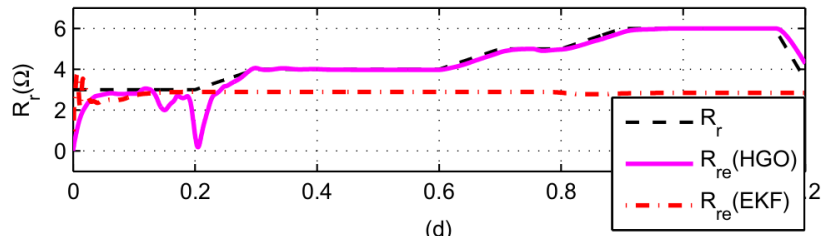

(d)

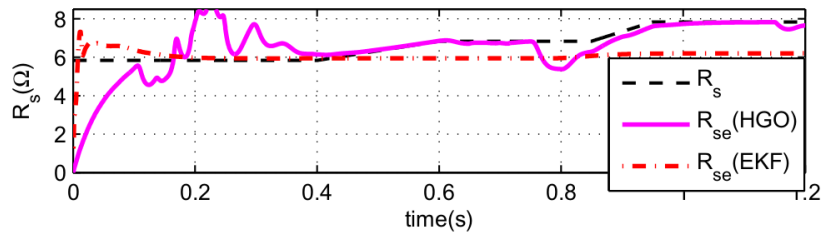

Fig. 2. Open loop estimation: comparison between adaptive HGO and EKF. 


$$
\left\{\begin{array}{l}
z_{1}^{1}=\hat{\omega}, \\
z_{2}^{1}=\|\widehat{\Psi}\|^{2}=\widehat{\Psi}_{\alpha r}^{2}+\widehat{\Psi}_{\beta r}^{2} \\
z_{1}^{2}=\frac{p M}{\widehat{L}_{r}} \hat{l}^{T} J_{2} \widehat{\Psi} \\
z_{2}^{2}=\hat{\imath}^{T} \widehat{\Psi} \\
\xi=\arctan \left(\widehat{\Psi}_{\beta r}\right)
\end{array}\right.
$$

Thus, system (2) can be rewritten in the new coordinates as follows:

$$
\left\{\begin{array}{l}
\dot{z}^{1}=A_{1}\left(\hat{\alpha}_{r}\right) z^{2}+\varphi^{1}\left(z^{1}, \hat{\alpha}_{r}, \widehat{T}_{L}\right) \\
\dot{z}^{2}=b\left(z, \xi, \hat{\alpha}_{r}\right) u+g\left(z, \hat{\alpha}_{r}, \hat{\alpha}_{s}\right) \\
\dot{\xi}=p z_{1}^{1}+\frac{\widehat{L}_{r} \hat{r}_{r}}{p} z_{1}^{2} \\
z_{2}^{1} \\
y=z^{1}
\end{array}\right.
$$

where $z=\left[z^{1^{T}}, z^{2^{T}}\right]^{T} \in \mathbb{R}^{4}$ is the state vector, $y \in \mathbb{R}^{2}$ is the measured output, and $u \in \mathcal{U} \in \mathbb{R}^{2}$.

$$
\begin{aligned}
& A_{1}\left(\hat{\alpha}_{r}\right)=\left(\begin{array}{cc}
\frac{1}{J} & 0 \\
0 & 2 M \hat{\alpha}_{r}
\end{array}\right), \varphi^{1}\left(z^{1}, \hat{\alpha}_{r}, \widehat{T}_{L}\right)=\left[\frac{-1}{J} \widehat{T}_{L},-2 \hat{\alpha}_{r} z_{2}^{1}\right]^{T} \\
& b\left(z, \xi, \hat{\alpha}_{r}\right)=\frac{1}{\sigma \widehat{L}_{s}} \sqrt{z_{2}^{1}}\left(\begin{array}{cc}
-p M \hat{\alpha}_{r} \sin (\xi) & \frac{p M}{\widehat{L}_{r}} \cos (\xi) \\
\cos (\xi) & \sin (\xi)
\end{array}\right) \\
& g\left(z, \hat{\alpha}_{r}, \hat{\alpha}_{s}\right)=\left[g_{1}\left(z, \hat{\alpha}_{r}, \hat{\alpha}_{s}\right) g_{2}\left(z, \hat{\alpha}_{r}, \hat{\alpha}_{s}\right)\right]^{T} \\
& g_{1}\left(z, \hat{\alpha}_{r}, \hat{\alpha}_{s}\right)=\frac{-\mu p^{2} M}{\widehat{L}_{r}} z_{1}^{1} z_{2}^{1}-\left(\gamma\left(\hat{\alpha}_{s}\right)+\hat{\alpha}_{r}\right) z_{1}^{2}-\frac{p^{2} M}{\widehat{L}_{r}} z_{1}^{1} z_{2}^{2} \\
& g_{2}\left(z, \hat{\alpha}_{r}, \hat{\alpha}_{s}\right)=\mu \hat{\alpha}_{r} z_{2}^{1}+\frac{\widehat{L}_{r}}{M} z_{1}^{1} z_{2}^{2}-\left(\gamma\left(\hat{\alpha}_{s}\right)+\hat{\alpha}_{r}\right) z_{2}^{2}+M \hat{\alpha}_{r} \frac{1}{z_{2}^{1}}\left(\left(z_{2}^{2}\right)^{2}+\left(\frac{\widehat{L}_{r}}{p M} z_{1}^{2}\right)^{2}\right)
\end{aligned}
$$

Let $z_{d}=\left[\begin{array}{ll}z_{d}^{T} & z_{d}^{2^{T}}\end{array}\right]^{T}$ with $z_{d}^{1}=\left[\begin{array}{ll}z_{d 1}^{1} & z_{d 2}^{1}\end{array}\right]^{T}$ be the reference trajectory vector corresponding to the mechanical speed and the square norm of the rotor flux.

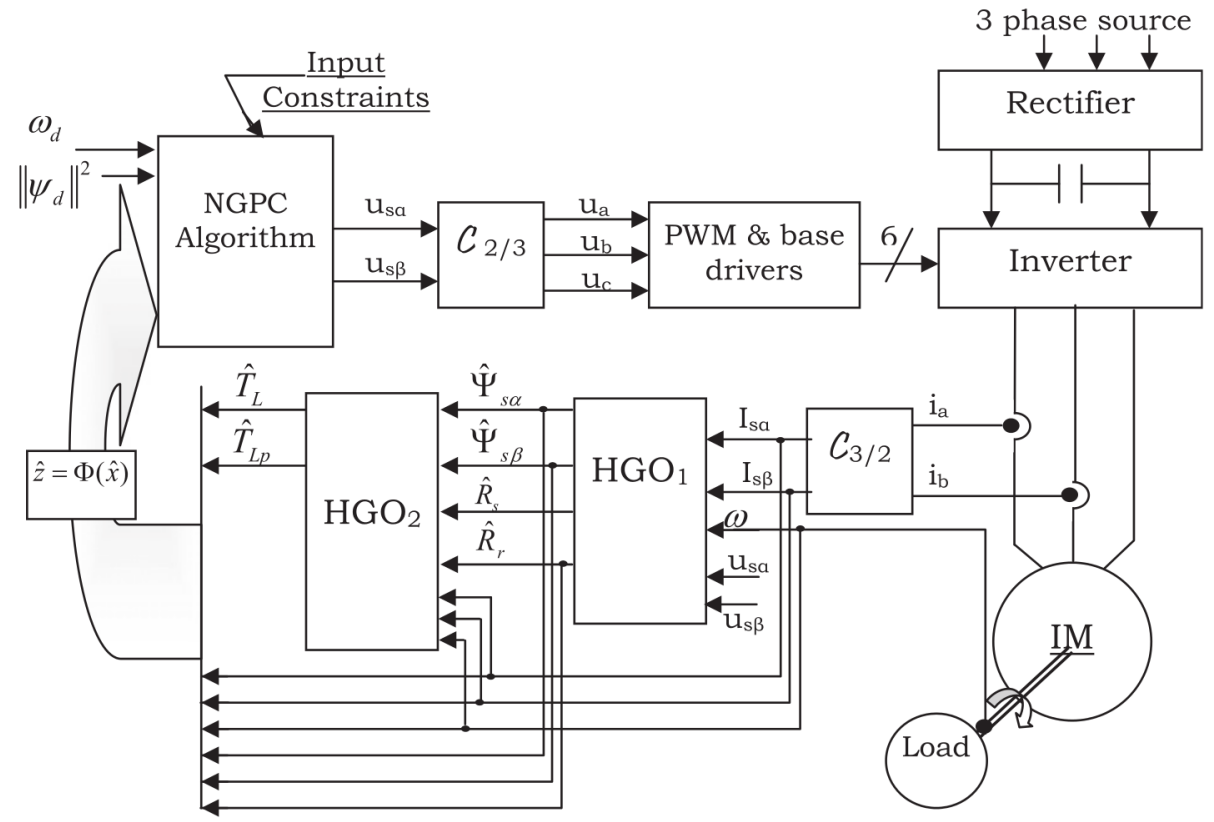

Fig. 3. Block diagrams of the output feedback predictive controller. 
The whole scheme of the control induction machine is depicted in Fig. 3. Mainly, after using the two-stage of HGO in order to estimate conjointly parameters and state, these lasts are incorporated in the predictive controller which inherently allows to take into account input constraints. $\mathcal{C}_{3 / 2}$ and $\mathcal{C}_{2 / 3}$ represent respectively direct and inverse Clarke transformations.

In the numerical simulation, we propose a trapezoidal smooth profile for speed reference trajectory, while reference corresponding to the square norm of the flux starts from zero and grow up until $t=0.1 \mathrm{~s}$ to a constant value which is equal to its rated value. Sensitivity of our control algorithm towards the motor resistances and the load torque mismatch is tested with an imposed scenario for a reasonable variation of these parameters. The solution of predictive control law arises from the QP formulation given by (18) with $T_{p}=5 \mathrm{~ms}$. It inherently incorporates stator voltage constraints such as: $A_{c}=\left[I_{2}-I_{2}\right]^{T}$ and

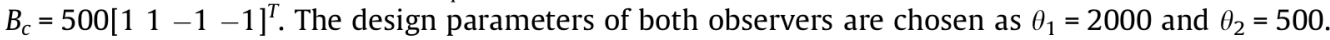

All simulation results are depicted in Figs. 4 and 5. Dynamic behavior of the output: rotor speed and flux modulus are illustrated in Fig. 4 graphs (a) and (b); while graphs (c), (d) and (e) of the same figure shows respectively the estimation of the load torque and both rotor and stator resistances. As it can be seen in graphs (a) and (b), the decoupling target of mechanical speed and rotor flux modulus is achieved through the proposed predictive controller even for low operating speed, so that its trajectories can be independently controlled. Moreover, we remark that the system outputs can track well the reference signals regardless of load torque and both resistances mismatch. In particular, with $100 \%$ variations of $R_{r}$ and $50 \%$ of $R_{\mathrm{S}}$ we have recorded a tracking velocity error less than two percent $(2 \%)$ and a tracking flux error less than ten percent $(10 \%)$. In the other hand and reported to the graphs (c)-(e), negligible estimation errors are shown in steady state operations while some fluctuations appear in the transient stages. Such behavior is explained as follows: when permanent regime is established (in steady state) the annulation of the angular speed dynamics $(\dot{\omega}=0)$ involves an annulation of the term of uncertainties $\varepsilon^{2}\left(\varepsilon^{2}=0\right)$; and by referring to (4), an asymptotic convergence of the estimation error is achieved. In contrast, the little bounded error seen when $\omega(t)$ changes level, is caused by the fact that $\varepsilon^{2} \neq 0$. One other drawback is that the proposed observer remains very sensitive to the noise measurements. So, the tuning of the design parameters $\theta_{1}$ and $\theta_{2}$ have required to satisfy a trade-off between the convergence of the estimation error and the objective of filtering noise.

(a)

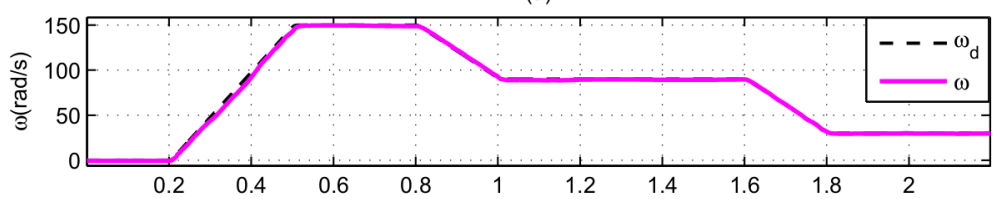

(b)

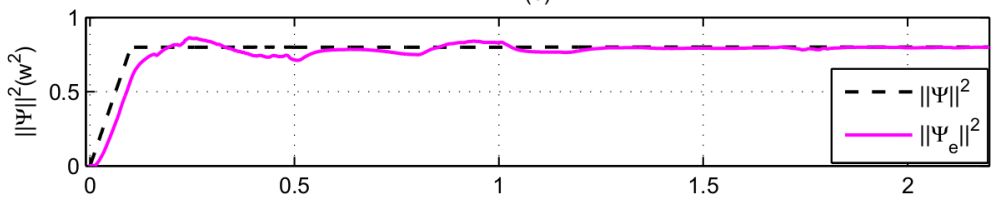

(c)

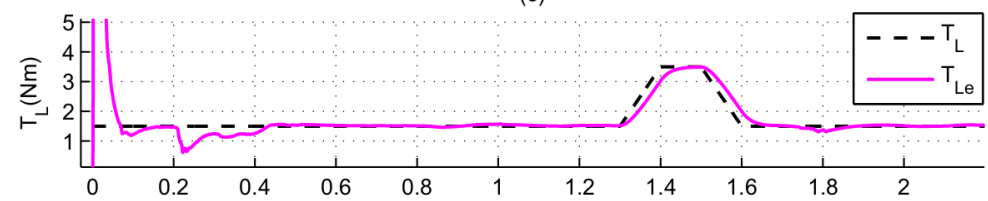

(d)

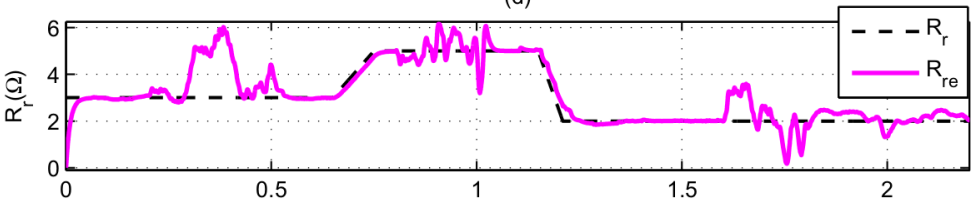

(e)

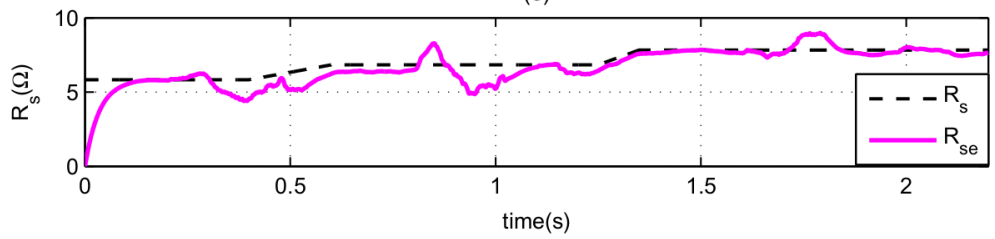

Fig. 4. Tracking performance and estimated variables in the closed loop. 

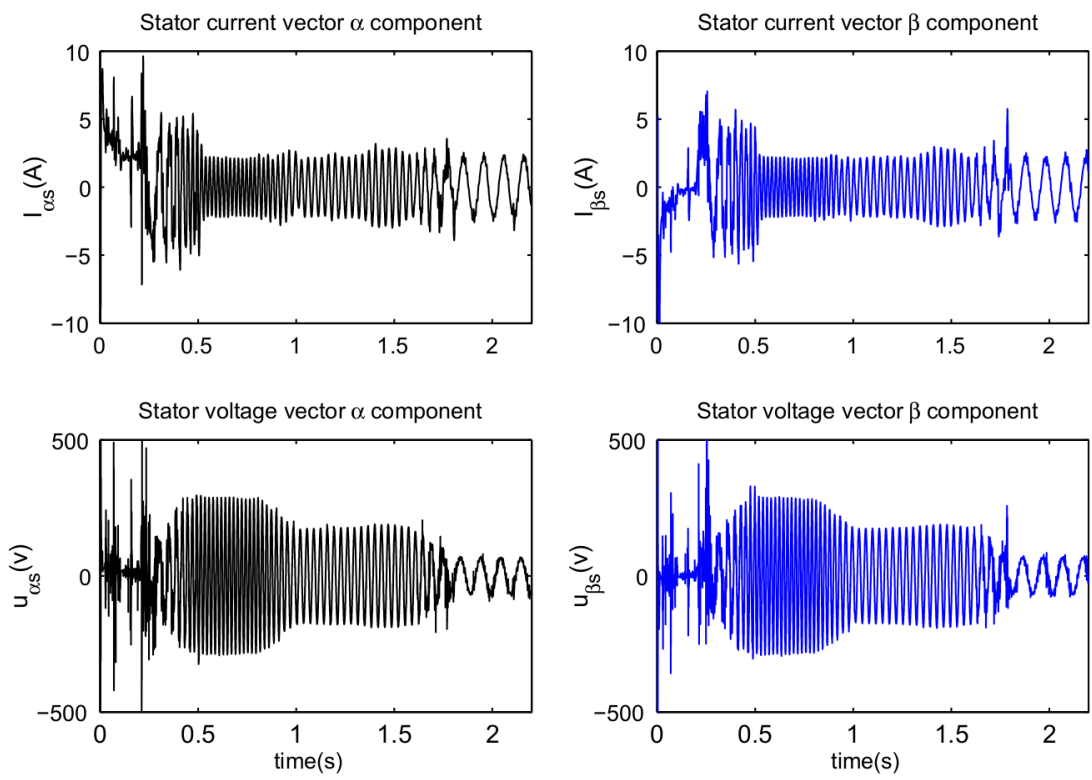

Fig. 5. $(\alpha, \beta)$ Components of stator current and voltage vectors.

Finally, the resulting $(\alpha, \beta)$ components of stator current and voltage vectors are reported in Fig. 5. Control voltages stay in the predefined bounds that helps to avoid peaking phenomenon from the system states. It is notable that the control effort can be reduced by increasing the predictive horizon, which leads, by opposite, to decrease the flux tracking performances.

\section{Conclusion}

In this paper, a constrained output feedback predictive controller is proposed as a novel approach for induction motor drive systems. The admissible tracking objective is originally obtained with acceptable performances in general. Precisely, despite of external disturbance (load torque variation) and parameters uncertainties (motor resistances mismatch), the decoupling target of mechanical speed and rotor flux modulus is almost achieved, even for low operating speed. There are two inherent characteristics that strengthen our design. On the one hand, the controller integrates cascade observers which allow recovering the unavailable states such as load torque and rotor flux, and adjusting the critical parameters such as rotor and stator resistances. On the other hand, our control algorithm incorporates input constraints which lead to isolate the peaking of estimates that usually presents a challenge in the observer-controller combination. The simulation results confirm that the conjoint estimation of states and parameters is asymptotically achieved in the steady state, and only a little bounded error is recorded at the transient. So, in order to emphasize our design for high performance applications, the proposed control scheme should be soon implemented in real time around the Dspace 1104 board. Further work will also concern the integration of other constraints in the control law formulation (i.e. stator currents, settling time, etc.) and the optimization of the computational burden.

\section{References}

[1] Barut M, Bogosyan MGS. Switching ekf technique for rotor and stator resistance estimation in speed sensorless control of ims. Energy Convers Manage 2007:48:3120-34.

[2] Ghanes M, Barbot J, Leon JD, Glumineau A. A robust output feedback controller of the induction motor drives: new design and experimental validation. Int J Control 2010;83:484-97.

[3] Kenne G, Tarek Ab, Lamnabhi-Lagarriguec F, Arzande A. Nonlinear systems time-varying parameter estimation: application to induction motors. Electric Power Syst Res 2008;78:1881-8.

[4] Marino R, Tomei P, Verrelli C. An adaptive tracking control from current measurements for induction motors with uncertain load torque and rotor resistance. Automatica 2008:44:2593-9.

[5] Sanchez PR, Cerradab AG, Batllea V. Rotor-resistance estimation for induction machines with indirect-field orientation. Control Eng Practice 2007:48:1119-33.

[6] Akin E, Kaya M, Karakose M. A robust integrator algorithm with genetic based fuzzy controller feedback for direct vector control. Comput Electric Eng 2003:3:379-94.

[7] Azza HB, Jemli M, Boussak M, Gossa M. High performance sensorless speed vector control of spim drives with on-line stator resistance estimation. Simul Modell Practice Theory 2011;19:271-82.

[8] Beguenane R, Ouhrouche MA, Trzynadlowski AM. A new scheme for sensorless induction motor control drives operating in low speed region. Math Comput Simul 2006;71:109-20.

[9] Kojabadi HM. Active power and mras based rotor resistance identification of an im drive. Simul Modell Practice Theory 2009;17:376-89. 
[10] Krilshnan R, Bharadwaj A. A review of parameter sensitivity and adaptation in indirect vector controlled induction motor drive systems. IEEE Trans Power Electron 1991;6:695-703.

[11] Marino R, Tomei P, Peresada S. On-line stator and rotor resistance estimation for induction motors. IEEE Trans Cont Syst Techn 2000;8:570-9.

[12] Alexandru T, Besancon G. Simultaneous state and parameter estimation in asynchronous motors under sensorless speed control. In: ECC, Orlando USA; 2003. p. 5075-80.

[13] Lascu C, Boldea A, Blaabjerg F. Direct torque control of sensorless induction motor drives: a sliding-mode approach. IEEE Trans Indus Appl 2004;40:582-90.

[14] Ha IJ, Lee S. An online identification method for both stator and rotor resistances of induction motors without rotational transducers. IEEE Trans Ind Electron 2000;47:842-53.

[15] Barut M. Bi input-extended Kalman filter based estimation technique for speed-sensorless control of induction motors. Energy Convers Manage 2010;51:2032-40.

[16] Magni L, Raimondo DM, Allgöwer F, editors. Nonlinear model predictive control, Heidelberg; 2009.

[17] Findeisen R, Imsland L, Allgöwer F, Foss B. Output feedback stabilization of constrained systems with nonlinear predictive control. Int J Robust Nonlinear Control 2003;13:211-27.

[18] Chen W. Predictive control of general nonlinear systems using approximation. IEE Proc Cont Theory Appl 2004;151:137-43.

[19] Chen W, Ballance D, Gawthrop P. Optimal control of nonlinear systems: a predictive control approach. Automatica 2003;39:633-41.

[20] Farza M, M'Saad M, Sekher M. A set of observers for a class of nonlinear systems. In: 16th IFAC W.C., Czech R.; 2005. p. 4-8.

[21] Farza M, M'Saad M, Rossignol L. Observer design for a class of mimo nonlinear systems. Automatica 2004;40:135-43.

[22] Saïd SH, Mimouni M, M’Sahli F, Farza M. High gain observer based on-line rotor and stator resistances estimation for ims. Simul Modell Practice Theory $2011 ; 19: 1518-29$.

[23] Atassi A, Khalil H. A separation principle for the stabilization of a class of nonlinear systems. IEEE Trans Automatic Control $2000 ; 44: 1672-87$.

[24] Spooner J, Maggiore M, Ordonez R, Passino KM. Stable adaptive control and estimation for nonlinear systems. John Wiley \& Sons; 2002. 